Discrete Comput Geom 34:295-312 (2005)

DOI: $10.1007 / \mathrm{s} 00454-005-1167-1$

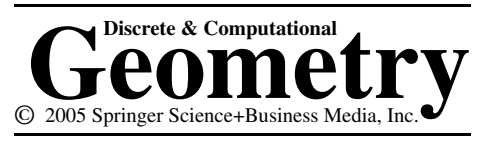

\title{
Dissection of a Triangle into Similar Triangles
}

\author{
Andrzej Zak \\ Faculty of Applied Mathematics, AGH University of Science and Technology, \\ al. Mickiewicza 30, 30-059 Kraków, Poland \\ zakandrz@uci.agh.edu.pl
}

\begin{abstract}
We prove that the number of non-similar triangles $T$ which can be dissected into two, three or five similar non-right triangles is equal to zero, one and nine, respectively. We find all these triangles. Moreover, every triangle can be dissected into $n$ similar triangles whenever $n=4$ or $n \geq 6$. In the last section we allow dissections into right-triangles but we add another restriction. We prove that in any perfect, prime and simplicial dissection into at least three tiles, the tiles must have one of only three possible shapes.
\end{abstract}

\section{Introduction}

Let $P$ and $P^{\prime}$ be polygons in the Euclidean plane. A dissection (tiling) of $P$ into $P^{\prime}$ is a decomposition of $P$ into finitely many, internally disjoint polygons $P_{1}^{\prime}, \ldots, P_{n}^{\prime}(n \geq 2)$ such that all of the $P_{i}^{\prime}$ are similar to $P^{\prime}$. A dissection is perfect if the $P_{i}^{\prime}$ are pairwise incongruent. The perfect decomposition of rectangles and squares into squares has been extensively studied (see [1] and also [3] for a detailed account of the history of this problem). The first example of a dissection of a rectangle into nine pairwise incongruent squares was given by Moron [7] in 1925. In 1939 Sprague [10] found the perfect squaring of a square into 55 tiles (see also [2]). Less is known about dissections of polygons other than the square. Tutte [11] proved that an equilateral triangle has no perfect dissections into smaller equilateral triangles. In 1991 Kaiser [5] observed that every non-equilateral triangle has a perfect dissection into at least six or eight tiles similar to it. His elementary construction is shown in Fig. 1. Laczkovich [6] showed that the number of non-similar triangles $\Delta$ such that a non-equilateral triangle $T$ has a dissection into $\Delta$, is at most six (he also showed that for the equilateral triangle $T$ there are infinitely many such $\triangle$ ).

In this paper we consider the following question: for every $n(n \geq 2)$ find those triangles $T$ that can be dissected into $n$ similar non-right triangles. We denote by $f(n)$ the number of such $T$ and by $g(n)$ the number of non-right triangles that have a dissection 


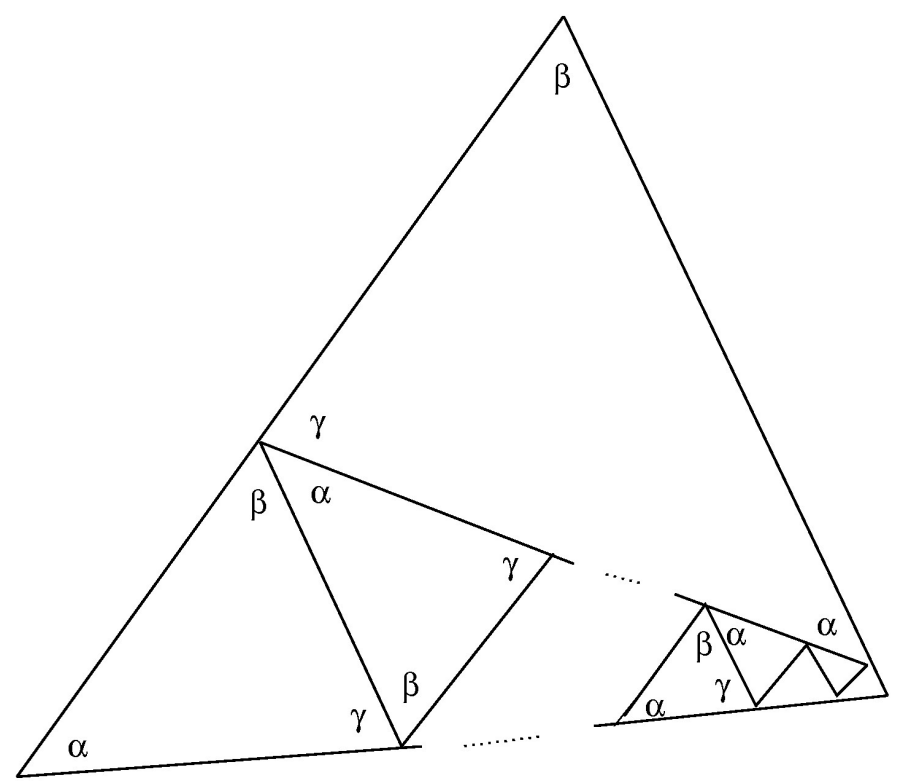

Fig. 1. Dissection into an even number of tiles.

into $n$ parts similar to the original. We prove that

- $f(2)=g(2)=0($ see Corollary 1$)$

- $f(3)=1$ and $g(3)=0$ (see Theorem 1 or [4]),

- $f(n)=g(n)=\infty$ whenever $n=4$ or $n \geq 6$ (see Fig. 1 if $n$ is even; to obtain the remaining $n$ 's dissect one tile into four parts; see also [4]),

- $f(5)=9$ and $g(5)=1$ (see Figs. 2-10 and Theorem 3).

The reason why we allow only non-right tiles is that every right or isosceles triangle can be easily dissected into an arbitrary number of similar right triangles. Moreover, in every decomposition of $T$ into similar right triangles, $T$ is right or isosceles (this follows straightforwardly from Theorems 4.1 and 5.1 to be found in the paper of Laczkovich [6]).

We can also conclude that five is the least number of tiles in any perfect tiling of $T$ into $\Delta$, whenever $\Delta$ is non-right. There are only two triangles, shown in Figs. 2 and 8, which can be dissected in this way. Moreover, no non-right triangle $T$ has a perfect dissection into less than six tiles similar to it. In Fig. 15 we present a perfect decomposition into six triangles similar to the original.

We want to point out that our question is related to the following problem, partly solved in [6] and [8], raised by Paul Erdös [9, Problem 6.7, p. 46]: find those integers $n$ for which there are triangles $T$ and $\triangle$ such that $T$ can be dissected into $n$ triangles congruent to $\triangle$.

In the last section we consider some special dissections. We call a dissection prime if no $k$ tiles, $2 \leq k \leq n-1$, form a triangle similar to them. We prove that in any perfect, prime and simplicial (i.e. the intersection of two distinct triangles is either empty or a vertex or an edge of both of them) tiling into at least three tiles, the tiles have to be 


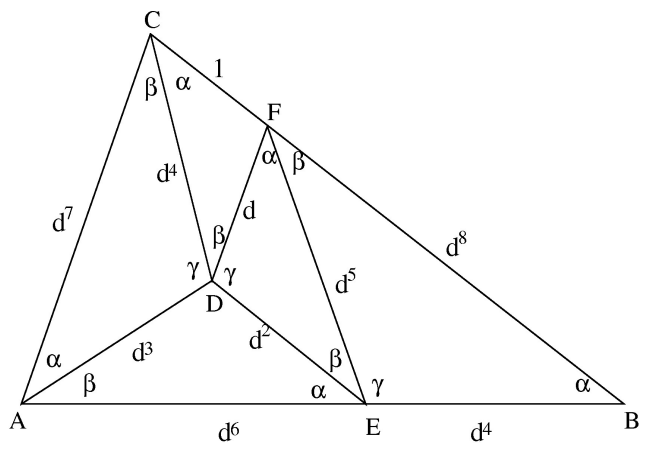

Fig. 2. Dissection from Lemma 1 , case $1\left(d^{6}-d^{2}-1=0\right)$.

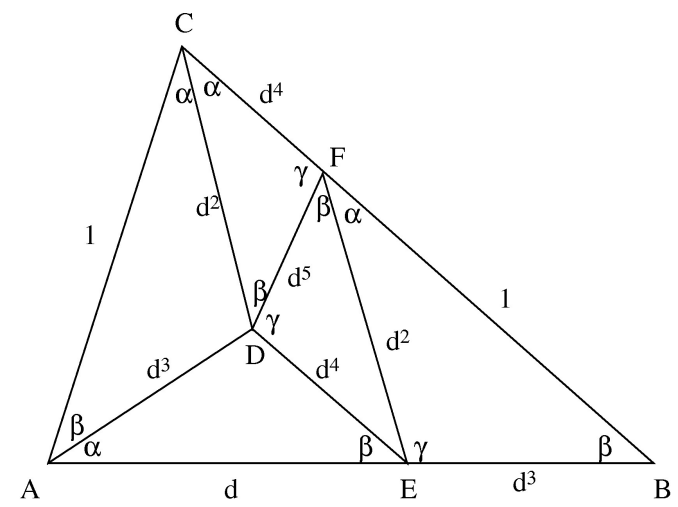

Fig. 3. Dissection from Lemma 1 , case $3\left(d^{6}+d^{2}-1=0\right)$.

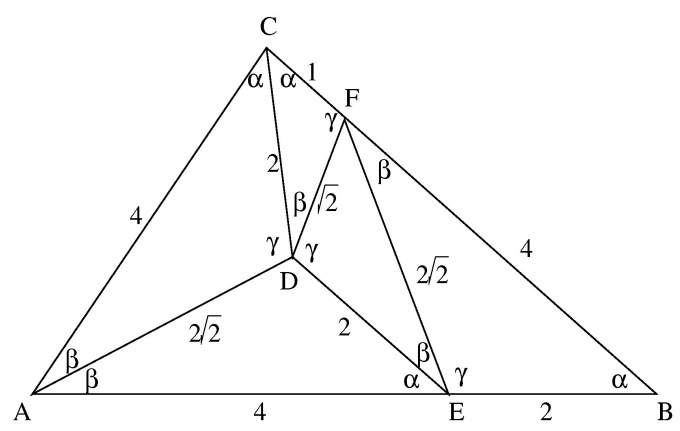

Fig. 4. Dissection from Lemma 1, case 2. 


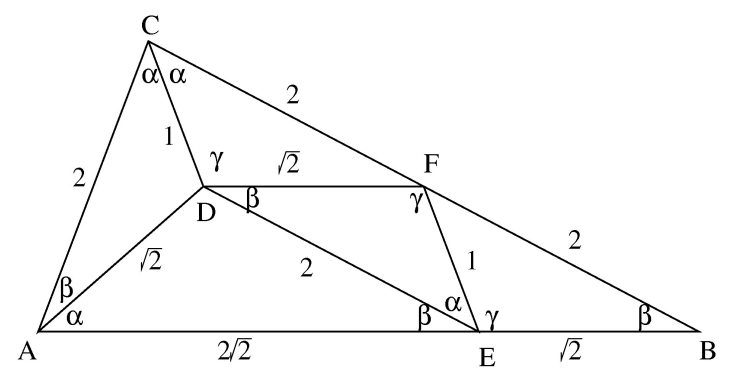

Fig. 5. Dissection from Lemma 1, case 2 .

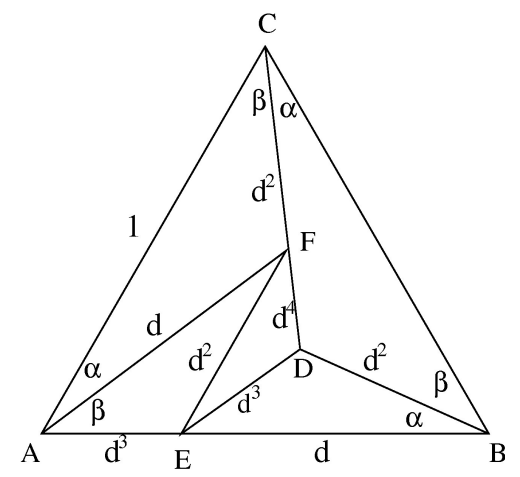

Fig. 6. Dissection from Lemma 2 , case (a) $\left(d^{3}+d-1=0\right)$.

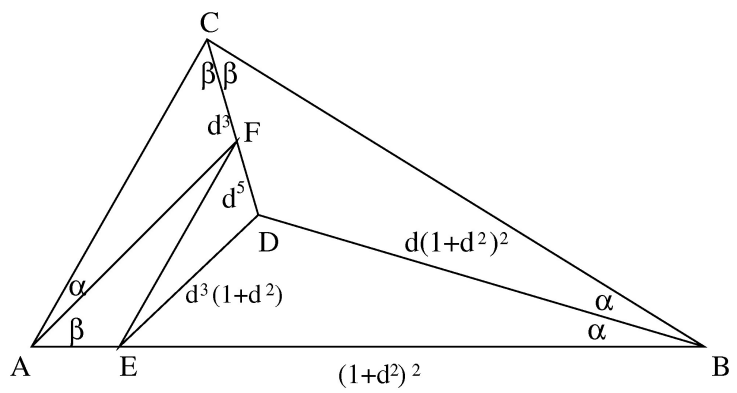

Fig. 7. Dissection from Lemma 2 , case (b) $\left(3 d^{6}+2 d^{4}-d^{2}-1=0\right)$. 


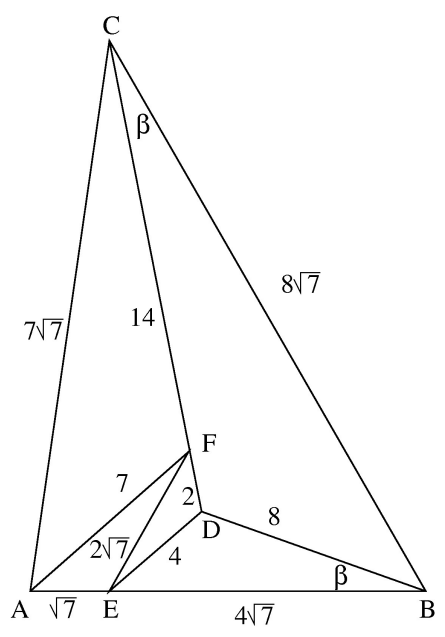

Fig. 8. Dissection from Lemma 2, case (b).

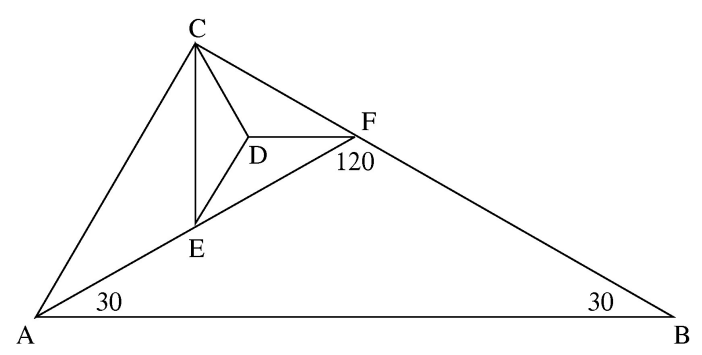

Fig. 9. Illustrating Theorem 3 when (**) occurs.

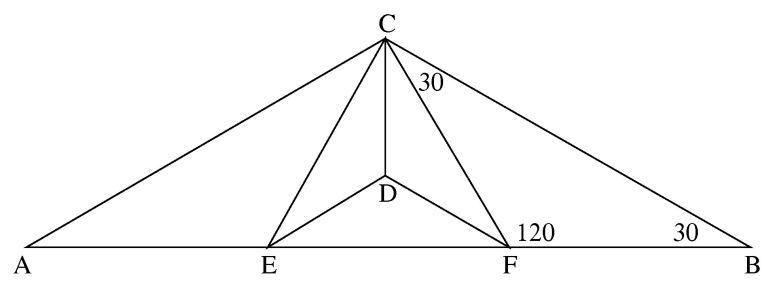

Fig. 10. Illustrating Theorem 3 when $(* *)$ occurs. 
one of three types given in Theorem 5. We add those supplementary restrictions in view to exclude all trivial dissections. In exchange we can allow decompositions into right triangles.

\section{Notation and Some Preparatory Remarks}

We sometime mean by the word dissection a graph obtained as a result of a dissection of a $k$-gon into $n(n \geq 2) l$-gons. Let $v$ and $e$ denote the number of its vertices and edges, respectively. We distinguish two sets among boundary vertices:

$V_{2}$-set of vertices of a given $k$-gon,

$V_{\mathrm{b}}$ - set of vertices lying on the boundary of a given $k$-gon, but different from its vertices.

Let $v_{2}$ and $v_{\mathrm{b}}$ denote the cardinalities of the above sets. Thus, $v_{2}=k$.

Similarly we distinguish two sets among internal vertices:

$V_{4}$-set of vertices which are internal points of sides of $l$-gons,

$V_{3}$-remaining vertices lying in the interior of a given $k$-gon.

Let $v_{4}$ and $v_{3}$ denote the cardinalities of those sets.

The above notation is justified in Proposition 2. Note that for a simplicial dissection $v_{4}=0$.

Summing the angles of $n l$-gons we obtain

$$
n(l-2) \cdot \pi=(k-2) \cdot \pi+v_{\mathrm{b}} \cdot \pi+v_{3} \cdot 2 \pi+v_{4} \cdot \pi .
$$

Thus

$$
v_{\mathrm{b}}+2 v_{3}+v_{4}=n(l-2)-k+2
$$

and, for $k=l=3$,

$$
v_{\mathrm{b}}+2 v_{3}+v_{4}=n-1 .
$$

Using Euler's formula $e=n+v-1=n+v_{2}+v_{3}+v_{4}+v_{\mathrm{b}}-1$ and formula (1) we obtain

$$
e=n(l-1)+1-v_{3}
$$

and, for $k=l=3$,

$$
e=2 n+1-v_{3}
$$

Proposition 1. A dissection of any polygon $P$ into a non-right triangle $\triangle$ cannot contain two angles which form a straight angle.

Proof. This is because the sum of two angles (not necessary different) of a non-right triangle is always different from $\pi$.

Corollary 1. No triangle can be decomposed into two similar non-right triangles. 
Let us recall the following definition. The degree of a vertex $x, \operatorname{deg} x$, is the number of edges incident to it, in other words the number of edges with $x$ as an endpoint.

Proposition 2. Every dissection of a polygon P into a non-right triangle $\triangle$ satisfies the following conditions:

1. if $x \in V_{2}$ then $\operatorname{deg} x \geq 2$,

2. if $x \in V_{3}$ then $\operatorname{deg} x \geq 3$,

3. if $x \in V_{4}$ or $x \in V_{\mathrm{b}}$ then $\operatorname{deg} x \geq 4$.

Proof. Properties 1 and 2 are obvious, and property 3 results from Proposition 1.

Proposition 3. If a dissection of a triangle $T$ into a non-right triangle $\triangle$ does not contain internal vertices $\left(v_{3}+v_{4}=0\right)$ then $v_{\mathrm{b}}=3$.

Proof. First note that no vertex $x \in V_{\mathrm{b}}$ can be directly connected (by edges) with two vertices lying on the same side of $T$ (including one of its end vertices). For in this case one of these two vertices must be directly connected with another vertex lying on the same side as $x$ (because $\operatorname{deg} x \geq 4$ if $x \in V_{\mathrm{b}}$ ). Moreover, this new vertex must be directly connected with yet another one lying on the same side of $T$ as the latter, etc. This operation has no end. Therefore, every vertex from the set $V_{\mathrm{b}}$ must be connected by edges with exactly two vertices lying on different sides of $T$. That means that $v_{\mathrm{b}}=3$.

Proposition 4. Suppose that a triangle T has been decomposed into a non-right triangle. If $v_{3}=0$ and $v_{4}=1$ then $v_{\mathrm{b}} \geq 4$.

Proof. Since $v_{3}=0$ then, by formula (4), $e=2 n+1$. On the other hand, by Proposition 2 and (2),

$$
e=\frac{\sum \operatorname{deg} x}{2} \geq \frac{4\left(v_{4}+v_{\mathrm{b}}\right)+2 v_{2}}{2}=\frac{4(n-1)+6}{2}=2 n+1 .
$$

Since the number of edges equals $2 n+1$, every vertex has its minimal possible degree given in Proposition 2 . Thus, the vertex from $V_{4}$ can be connected by edges only with vertices from the set $V_{\mathrm{b}}$. Since for each $x \in V_{4} \operatorname{deg} x \geq 4, v_{\mathrm{b}} \geq 4$.

To simplify further discussion we distinguish the following situation.

(**) There is a segment, different from any side of a triangle $T$, that connects one of the vertices of $T$ with the side opposite to it.

\section{Dissection into Three and Four Non-Right Tiles}

Theorem 1. Only an equilateral triangle $T$ can be dissected into three non-right similar triangles-this dissection is unique (Fig. 11). 


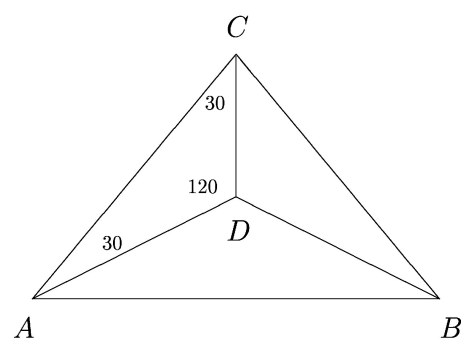

Fig. 11. Dissection into three tiles.

Proof. It is obvious that $v_{4}=0$. Hence, by formula (2), $v_{3}=1-v_{\mathrm{b}} / 2$. If $v_{\mathrm{b}}=2$ then $v_{3}=0$ but this is a contradiction to Proposition 3. Hence, $v_{3}=1$ and $v_{\mathrm{b}}=0$. Let $D \in V_{3}$. It must be connected with all vertices of $T$. Since the sum of any two angles around the vertex $D$ is greater than $\pi$, they must be equal. Thus, all three angles at $D$ are equal, hence are $\frac{2}{3} \pi$.

If the angles at one of the nodes of the triangle $T$ are equal, then the two tiles that share this node are congruent. Thus the third tile is isosceles (as well as the previous ones) with angles $\frac{2}{3} \pi$ and $\pi / 6$.

If at every node of $T$ two angles are different then, because their sum is $\pi / 3, T$ is equilateral. Thus the three tiles are congruent, hence are isosceles, contradicting the assumption that the angles are not equal.

Theorem 2. Every non-right triangle has a dissection into four similar non-right triangles-this dissection (Fig. 1) is unique with regard to the isomorphism of adequate graphs. Only one right triangle, the one with angles $\pi / 2, \pi / 3, \pi / 6$, has such a decomposition (Fig. 12).

Proof. Obviously $v_{4} \leq 1$, so formula (2) permits the following four cases:

1. $v_{4}=0, v_{\mathrm{b}}=1, v_{3}=1$,

2. $v_{4}=0, v_{\mathrm{b}}=3, v_{3}=0$,

3. $v_{4}=1, v_{\mathrm{b}}=0, v_{3}=1$,

4. $v_{4}=1, v_{\mathrm{b}}=2, v_{3}=0$.

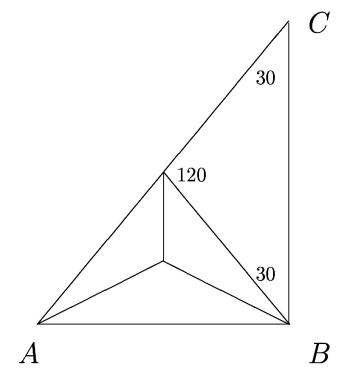

Fig. 12. Right triangle dissected into four tiles. 
In case 1 the vertex from $V_{\mathrm{b}}$ is connected by edges with at least four vertices. Thus, it must be connected by edges with all nodes of $T$. Hence, one of these edges divides $T$ into two triangles. Now we have to divide one of them into three non-right similar triangles. This is possible only in the way shown in Theorem 1 (Fig. 11). The whole situation is presented in Fig. 12.

Case 2 is a decomposition from Fig. 1. The necessity of marking these and no other edges arises from the proof of Proposition 3.

In case 3 the vertex from the set $V_{3}$ must be connected by segments with all vertices of $T$. Now we have three triangles so we have to divide one of them into two non-right similar triangles but this is not possible.

Case 4 is eliminated by Proposition 4.

It is now easy to check that

Corollary 2. There is no perfect tiling of a triangle into less than five non-right tiles.

\section{Dissection into Five Non-Right Tiles}

Lemma 1. There are four non-similar triangles $T$ which can be dissected into nonright triangle $\triangle$ in the way that gives the graph isomorphic to the one from Fig. 13.

Proof. Let $\alpha, \beta, \gamma$ be angles of $\triangle$. In the proof we assume that $\alpha \leq \gamma$ and $\beta \leq \gamma$. Thus, three of the angles around the vertex $D$ must equal $\gamma$ (the largest) with $\gamma>\pi / 2$. Owing to symmetry we can assume that the fourth is $\beta$. Thus $\beta=2 \pi-3 \gamma$ and $\alpha=2 \gamma-\pi$, hence

$$
\left\{\begin{array}{l}
\sin \alpha=-2 \sin \gamma \cos \gamma, \\
\sin \beta=\sin \gamma\left(4 \sin ^{2} \gamma-3\right) .
\end{array}\right.
$$

We apply the law of sines (four times) to the tiles that form the quadrangle $A E F C$. Thus the product of the sines of the angles marked by "*" in Fig. 13 is equal to the product of

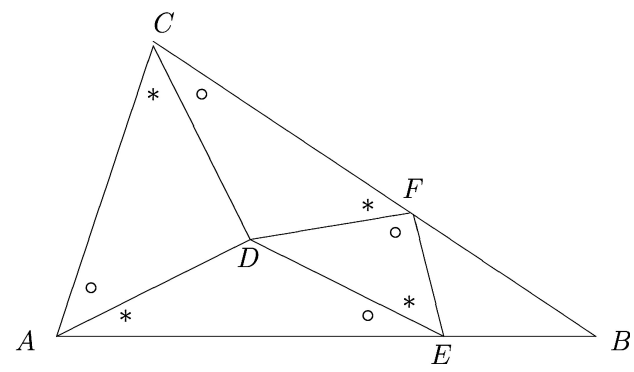

Fig. 13. Illustrating Lemma 1. 
the sines of the angles marked by "o" in this figure. Since the angles around the middle (vertex $D$ ) are $\beta$ and three times $\gamma$, the remaining ones are $\gamma$, three times $\beta$ and four times $\alpha$. Thus, we obtain four possible equations:

1. $\sin ^{4} \alpha=\sin ^{3} \beta \sin \gamma$,

2. $\sin ^{3} \alpha \sin \beta=\sin \alpha \sin ^{2} \beta \sin \gamma$,

3. $\sin ^{3} \alpha \sin \gamma=\sin \alpha \sin ^{3} \beta$,

4. $\sin ^{2} \alpha \sin ^{2} \beta=\sin ^{2} \alpha \sin \beta \sin \gamma$.

In case $1 \sin \gamma=\sqrt{a+3} / 2$ (i.e. $4 \sin ^{2} \gamma-3=a$ ) where $a$ is the unique real number that satisfies $a^{3}-a^{2}+2 a-1=0$. Moreover, only those vertices of the quadrangle $A E F C$ whose angle is $\alpha+\beta$ can be nodes of the original triangle $T$ (otherwise, the sum of angles of $T$ exceeds $\pi$ ). Hence, it can be easily checked that the angles of $T$ are $\alpha+\beta, \alpha+\beta, \alpha$ (Fig. 2).

In case $2 \sin \gamma=\sqrt{\frac{7}{8}}$. Checking that in this case the angles of $T$ are $2 \alpha, \alpha+\beta, \beta$ (Fig. 5) or $2 \alpha, 2 \beta, \alpha$ (Fig. 4) is left to the reader. Note, however, that the angles of $T$ cannot be $\alpha+\gamma, 2 \alpha, \alpha$ because then $\gamma=\frac{5}{9} \pi$ which is impossible for $\sin \gamma=\sqrt{\frac{7}{8}}$.

In case $3 \sin \gamma=\sqrt{a+3} / 2$ where $a$ is the unique real number that satisfies $a^{3}+a-$ $1=0$. In this case the angles of $T$ are $2 \alpha, \alpha+\beta, \beta$ (Fig. 3).

Case 4 can be excluded because then $\beta=\gamma=\pi / 2$ and $\alpha=0$.

Lemma 2. There are three non-similar triangles $T$ that can be dissected into non-right triangle $\triangle$ in a way that gives a graph isomorphic to the one from Fig. 14.

Proof. Let $\alpha, \beta, \gamma$ be angles of $\triangle$. In the proof we assume that $\alpha \leq \gamma$ and $\beta \leq \gamma$. Since there exists a vertex $x \in V_{3}$ with $\operatorname{deg} x=3$, all three angles at this vertex must equal $\frac{2}{3} \pi$, hence $\gamma=\frac{2}{3} \pi$ and $\alpha+\beta=\pi / 3$. Note also that only $\alpha$ and $\beta$ can be components of the angles of $T$. Without loss of generality we can assume that $\angle A C F=\beta$. Thus, after every possible distribution of $\alpha, \beta, \gamma$ we obtain that the angles of $T$ are equal to

(a) $\alpha+\beta, \alpha+\beta, \alpha+\beta$ or

(b) $\alpha+\beta, 2 \alpha, 2 \beta$

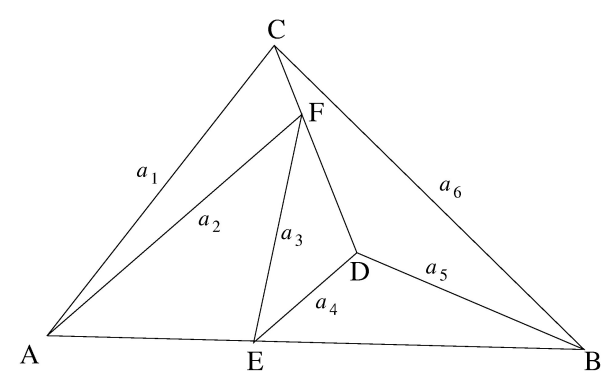

Fig. 14. Illustrating Lemma 2. 
We apply the law of sines to the five tiles and to $T$. Thus, in five small triangles

$$
\begin{aligned}
\frac{a_{1}}{\sin F} & =\frac{a_{2}}{\sin C}, & \frac{a_{2}}{\sin E}=\frac{a_{3}}{\sin A}, & \frac{a_{3}}{\sin D}=\frac{a_{4}}{\sin F}, \\
\frac{a_{4}}{\sin B}=\frac{a_{5}}{\sin E}, & \frac{a_{5}}{\sin C}=\frac{a_{6}}{\sin D} & &
\end{aligned}
$$

and in $T$,

$$
\frac{a_{1}}{\sin B}=\frac{a_{6}}{\sin A} .
$$

Therefore, in case (b), we obtain three possibilities

$$
a_{6}=a_{1} \frac{4 \sin ^{3} \beta}{3 \sin \alpha} \quad \text { and } \quad \frac{a_{1}}{\sin 2 \alpha}=\frac{a_{6}}{\sin (\alpha+\beta)}
$$

or

$$
a_{6}=a_{1} \frac{4 \sin ^{3} \alpha}{3 \sin \beta} \quad \text { and } \quad \frac{a_{1}}{\sin (\alpha+\beta)}=\frac{a_{6}}{\sin 2 \alpha}
$$

or

$$
a_{6}=a_{1} \frac{4}{3} \sin ^{2} \alpha \quad \text { and } \quad \frac{a_{1}}{\sin 2 \beta}=\frac{a_{6}}{\sin 2 \alpha} .
$$

Thus,

$$
16 \sin ^{3} \beta \cos \alpha=3 \sqrt{3}, \quad \alpha+\beta=\frac{\pi}{3}
$$

or

$$
3 \cos \alpha \sin \beta=\sqrt{3} \sin ^{2} \alpha, \quad \alpha+\beta=\frac{\pi}{3}
$$

or

$$
2 \sin \alpha \sin 2 \beta=3 \cos \alpha, \quad \alpha+\beta=\frac{\pi}{3} .
$$

In the first two equations the left sides are decreasing and the right sides are nondecreasing when $\alpha$ changes from 0 to $\pi / 3$. Hence, each of these equations has at most one solution, in fact exactly one. Adequate decompositions are in Figs. 7 and 8 . The third equation does not have any solution for

$$
2 \sin \alpha \sin 2 \beta<\sqrt{2}<\frac{3}{2}<3 \cos \alpha \quad \text { if } \quad \alpha \in\left(0, \frac{\pi}{4}\right]
$$

and

$$
2 \sin \alpha \sin 2 \beta<\frac{\sqrt{3}}{2}<\frac{3}{2}<3 \cos \alpha \quad \text { if } \quad \alpha \in\left(\frac{\pi}{4}, \frac{\pi}{3}\right) .
$$

In case (a) $T$ is equilateral. Although the shape of tiles does not affect the shape of $T$, we can make analogous calculations and obtain that at most one triangle $\Delta$ realizes this dissection. In fact, excactly one, as was shown in Fig. 6.

Theorem 3. There are nine non-similar triangles (shown in Figs. 2-10) that can be decomposed into five similar non-right triangles. 
Proof. Obviously $v_{4} \leq 1$. Thus, formula (2) and Proposition 3 permit the following four possibilities:

1. $v_{4}=1, v_{\mathrm{b}}=3, v_{3}=0$,

2. $v_{4}=1, v_{\mathrm{b}}=1, v_{3}=1$,

3. $v_{4}=0, v_{\mathrm{b}}=2, v_{3}=1$,

4. $v_{4}=0, v_{\mathrm{b}}=0, v_{3}=2$.

Case 1 is eliminated by Proposition 4.

Case 4 can also be easily excluded. Let $x, y \in V_{3}$. Euler's formula gives $e=9$, so the dissection contains six "internal" edges. Thus, $\operatorname{deg} x=4$ and $\operatorname{deg} y=3$. Hence, the three angles at the vertex $y$ are equal $\frac{2}{3} \pi$. However, it is impossible now to distribute proper values for four angles that form a full angle around the vertex $x$.

If (**) occurs, then the adequate segment divides $T$ into two triangles. Thus, we have to divide one of them into four similar, non-right triangles. That could be done in two ways as mentioned in Theorem 2. However, here only the one from Fig. 12 is adequate—otherwise a vertex $x \in V_{\mathrm{b}}$ with $\operatorname{deg} x=3$ occurs. The triangle from Fig. 12 can be placed within $T$ in two ways giving the two dissections shown in Figs. 9 and 10.

If $(* *)$ does not occur, then in case 3 we obtain the graph from Fig. 13, hence, by Lemma 1, we obtain four decompositions (Figs. 2-5). In case 2 the vertex from the set $V_{\mathrm{b}}$ must be connected by edges with two vertices-one from $V_{3}$ and one from $V_{4}$. The latter must lie on the segment connecting the vertex from $V_{3}$ with one of the vertices of $T$. Hence, the graph of this tiling is the one from Fig. 14. Thus, by Lemma 2, we obtain the next three dissections.

\section{Some Special Decompositions}

The construction from Fig. 1 shows that every triangle $T$ has a dissection into any even, greater than or equal to four number of tiles. Thus, by dividing one tile into four pieces we obtain a dissection into any odd, greater than or equal to seven number of tiles. The dissection from Fig. 1 is mostly perfect (see [5]). Moreover, by dividing the smallest tile in a perfect way we obtain again a perfect dissection.

To eliminate all trivial dissections we consider only perfect, prime and simplicial decompositions into at least three tiles. In exchange we allow right tiles. Theorem 5 shows that these decompositions are very rare. Figures 2 and 15 present two examples. In particular, Fig. 15 presents a nice perfect, prime and simplicial dissection into tiles similar to the original. Note also that by sticking on a new tile to the triangle from Fig. 2 we obtain a perfect and prime (but not simplicial) dissection of this triangle into six tiles (see Fig. 16). By repetition of this operation we can obtain a perfect and prime dissection of this triangle into any greater than or equal to five number of tiles.

Proposition 5. In every perfect, prime and simplicial dissection of a triangle $T$ into at least three similar triangles $\operatorname{deg} x \geq 4$ for each vertex $x \in V_{\mathrm{b}}$. 


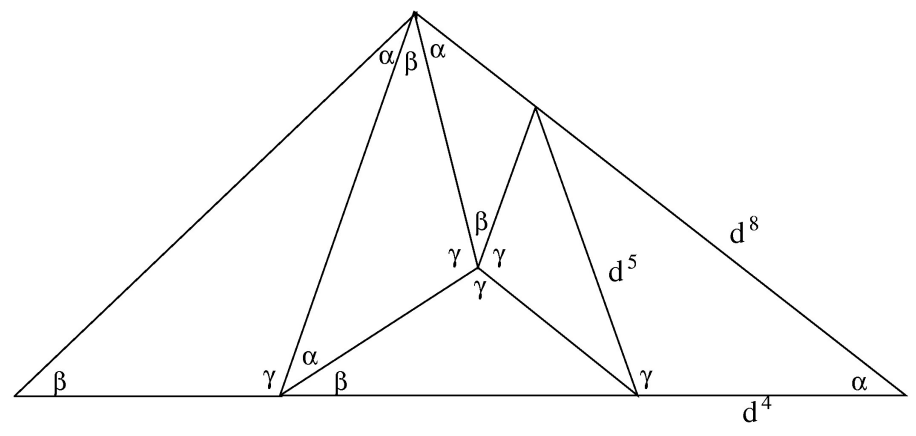

Fig. 15. Perfect, prime and simplicial dissection $\left(d^{6}-d^{2}-1=0\right)$.

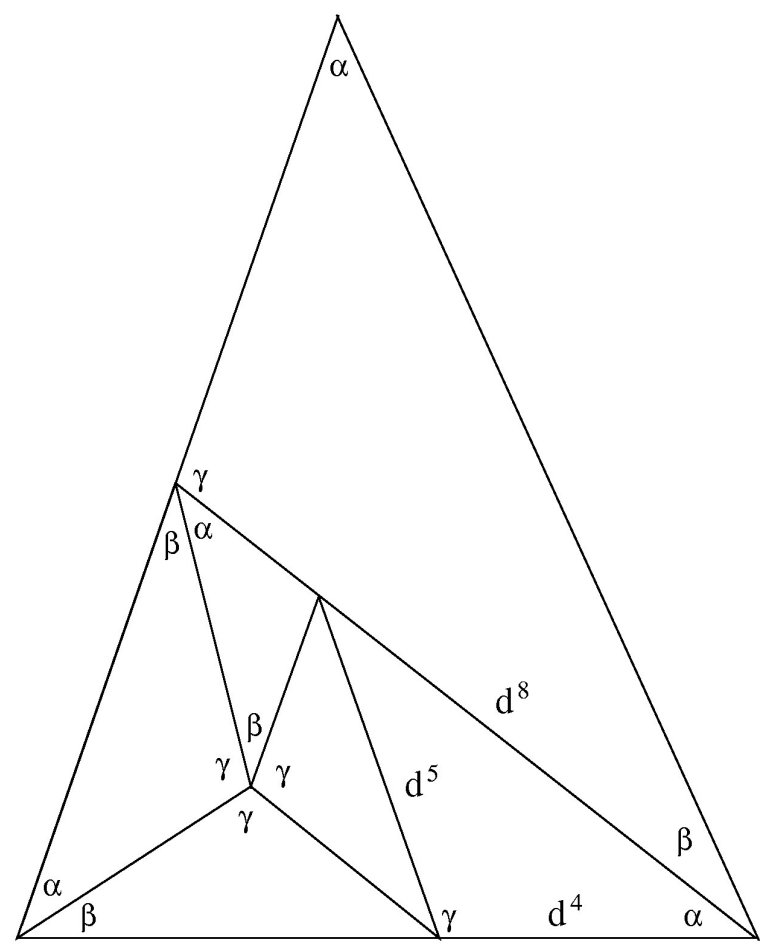

Fig. 16. Perfect and prime dissection $\left(d^{6}-d^{2}-1=0\right)$. 
Proof. Suppose that $\operatorname{deg} x=3$ for some $x \in V_{\mathrm{b}}$. Thus, by Proposition 2, the tiles are right triangles. Since a dissection is simplicial then two tiles have a common leg. Therefore they are congruent or form a triangle similar to them. Anyway the dissection cannot be perfect as well as prime.

Lemma 3. In every perfect, prime and simplicial dissection of a triangle $T$ into at least three similar triangles one of the following statements is true:

1. there exists a vertex $x \in V_{3}$ such that $\operatorname{deg} x \leq 5$,

2. set $V_{3}$ is empty,

3. for all $x \in V_{3}, \operatorname{deg} x=6$, for all $x \in V_{\mathrm{b}}, \operatorname{deg} x=4$ and for all $x \in V_{2}, \operatorname{deg} x=2$.

Proof. By formula (4)

$$
\begin{aligned}
4 n+2-2 v_{3}=2 e & =\sum_{v \in V_{3}} \operatorname{deg} v+\sum_{v \in V_{\mathrm{b}}} \operatorname{deg} v+\sum_{v \in V_{2}} \operatorname{deg} v \\
& \geq \sum_{v \in V_{3}} \operatorname{deg} v+4 v_{\mathrm{b}}+6 .
\end{aligned}
$$

Applying formula (2) we obtain

$$
\sum_{v \in V_{3}} \operatorname{deg} v \leq 4 n+2-2 v_{3}-6-4 \cdot\left(n-1-2 v_{3}\right) .
$$

Thus,

$$
v_{3} \cdot \min _{v \in V_{3}} \operatorname{deg} v \leq 6 \cdot v_{3}
$$

and the equality holds if and only if for all $x \in V_{3}, \operatorname{deg} x=6$, for all $x \in V_{\mathrm{b}}, \operatorname{deg} x=4$ and for all $x \in V_{2}, \operatorname{deg} x=2$.

Theorem 4. In every perfect, prime and simplicial dissection of a triangle $T$ into at least three similar triangles there exists a vertex $x \in V_{3}$ such that $\operatorname{deg} x=4$ or $\operatorname{deg} x=5$.

Proof. If for some $x \in V_{3}, \operatorname{deg} x=3$ then, since the dissection is simplicial, we obtain a triangle dissected into three triangles as in Fig. 11. Hence tiles are congruent.

If $v_{3}=0$ then, since $\operatorname{deg} x \geq 4$ if $x \in V_{\mathrm{b}}$, we can repeat the proof of Proposition 3 and obtain that the dissection is the one from Fig. 1 with $n=4$. It is easy to check that it cannot be perfect.

Therefore, let us consider case 3 from the previous lemma. Assume that $\alpha, \beta, \gamma$ are the angles of each tile. Note that $\alpha, \beta$ and $\gamma$ must be pairwise different to have any chance of a perfect dissection. Thus, the distribution of angles $\alpha, \beta, \gamma$ at every vertex from $V_{\mathrm{b}}$ can be

(i) $1-1-1$ or

(ii) $3-0-0$ 


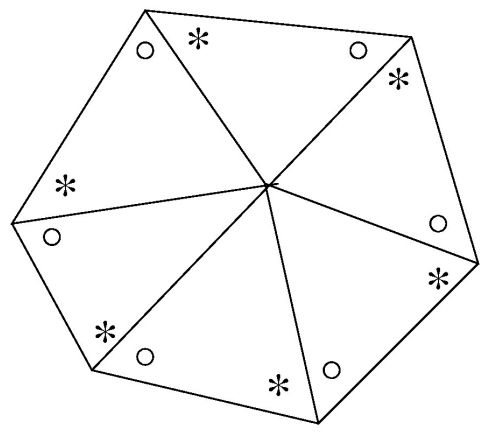

Fig. 17. Hexagon induced by $x \in V_{3}$ with $\operatorname{deg} x=6$.

and at every vertex from $V_{3}$
(a) $2-2-2$ or
(b) 3-3-0 or
(c) $4-1-1$ or
(d) $5-1-0$ or
(e) $6-0-0$

(with a suitable permutation of $\alpha, \beta, \gamma)$. Since the dissection is simplicial, six triangles which have a common node in some vertex $x \in V_{3}$ form a hexagon.

In case (b) all six angles marked by "*” in Fig. 17 or all six angles marked by "o" are equal, say they are equal $\gamma(\gamma=\pi / 3)$. Indeed, otherwise two adjacent triangles share an " $\alpha, \beta$-side", hence are congruent. Moreover, the angles at the middle are three times $\alpha$ and three times $\beta$. We apply the law of sines (six times) to this hexagon. Thus,

$$
\sin ^{6} \gamma=\sin ^{3} \alpha \sin ^{3} \beta, \quad \text { hence, } \quad \sin ^{2} \gamma=\sin \alpha \sin \beta .
$$

Thus,

$$
\frac{3}{4}=\sin \alpha \sin \left(\frac{2}{3} \pi-\alpha\right) .
$$

Checking that $\alpha=\pi / 3$ is left to the reader. Therefore, $\alpha=\beta=\gamma$ so a dissection cannot be perfect, hence case (b) can be excluded.

Assume now situation (ii) or (c) or (d) or (e). Since the number of angles $\alpha$ at some vertex $x \in V_{3} \cup V_{\mathrm{b}}$ is pretty large, there exists another vertex $y \in V_{3} \cup V_{\mathrm{b}}$ that compensates the total number of $\alpha$ 's. Thus the number of $\alpha$ 's at $y$ is less than two if $y \in V_{3}$ or equals zero if $y \in V_{\mathrm{b}}$. Indeed, otherwise the global number of $\alpha$ 's is greater than or equal to

$$
2+\sum_{v \in V_{3}} 2+\sum_{v \in V_{\mathrm{b}}} 1=2+2 v_{3}+v_{\mathrm{b}},
$$

which, by (2), is equal to $n+1$. This, however, is a contradiction because there are exactly $n \alpha$ 's, for there are $n$ tiles and $\alpha, \beta, \gamma$ are pairwise different. Therefore one of 
the following systems of equations holds:

$$
\begin{gathered}
{\left[\begin{array}{lll}
4 & 1 & 1 \\
p & q & r \\
2 & 2 & 2
\end{array}\right]\left[\begin{array}{l}
\alpha / \pi \\
\beta / \pi \\
\gamma / \pi
\end{array}\right]=\left[\begin{array}{l}
2 \\
2 \\
2
\end{array}\right] \text { or }\left[\begin{array}{lll}
5 & 1 & 0 \\
p & q & r \\
2 & 2 & 2
\end{array}\right]\left[\begin{array}{l}
\alpha / \pi \\
\beta / \pi \\
\gamma / \pi
\end{array}\right]=\left[\begin{array}{l}
2 \\
2 \\
2
\end{array}\right]} \\
\text { or }\left[\begin{array}{lll}
6 & 0 & 0 \\
p & q & r \\
2 & 2 & 2
\end{array}\right]\left[\begin{array}{l}
\alpha / \pi \\
\beta / \pi \\
\gamma / \pi
\end{array}\right]=\left[\begin{array}{l}
2 \\
2 \\
2
\end{array}\right],
\end{gathered}
$$

where $p \leq 1$ and $p+q+r=6$. Note that the all above-mentioned cases are included because cases (ii) and (e) are equivalent here. Since $\alpha=\beta=\gamma=\pi / 3$ is a solution of each of the above systems (a solution not being allowed for us) then at least one of those systems must have infinitely many solutions. Thus the adequate determinant equals zero. Hence also at least one of the determinants given below equals zero (otherwise the system in question has no solution). In the first case

$$
\operatorname{det}\left[\begin{array}{lll}
4 & 1 & 2 \\
p & q & 2 \\
2 & 2 & 2
\end{array}\right]=0 \quad \Leftrightarrow \quad 2 q+p=6
$$

Since $p \leq 1,(p, q, r)=(0,3,3)$ but this distribution of angles is not possible for us. In the second case

$$
\operatorname{det}\left[\begin{array}{lll}
5 & 1 & 2 \\
p & q & 2 \\
2 & 2 & 2
\end{array}\right]=0 \quad \Leftrightarrow \quad 3 q+p=8 .
$$

However, there is no pair $(p, q)$ with $p \leq 1$ which satisfies this condition. In the third case

$$
\operatorname{det}\left[\begin{array}{lll}
6 & 0 & 0 \\
p & q & r \\
2 & 2 & 2
\end{array}\right]=0 \Leftrightarrow q=r .
$$

Hence, again $(p, q, r)=(0,3,3)$ which is not possible here.

Therefore, a distribution of angles at every vertex $x$ is 2-2-2 if $x \in V_{3}$ and 1-11 if $x \in V_{\mathrm{b}}$. We consider a part of our dissection shown in Fig. 18. Without loss of generality we can assign the angles at the vertex $V$ by $\alpha, \beta, \gamma$ as in this figure. Suppose now that the angle $\angle V U X$ equals $\alpha$ (the assumption that it is equal to $\gamma$ leads to the analogous argumentation but then distribution of remaining angles is different). Hence, the remaining angles assigned by $\alpha, \beta$ or $\gamma$ are uniquely determined (if $\angle U Z Y$ does not equal $\gamma$, then $\triangle Y U Z$ is congruent with $\triangle X U Y$ or with $\triangle A V X)$. Since the angles around $U$ are $\alpha, \alpha, \beta, \beta, \gamma, \gamma$, the angles marked by " $*$ " coincide as well as the angles marked by "o". However, now two angles at the vertex $W \in V_{\mathrm{b}}$ are equal, hence, the distribution of angles at this vertex is not 1-1-1. Therefore, in case 3 , in each possible situation we obtained a contradiction with the assumption that a dissection is perfect. 


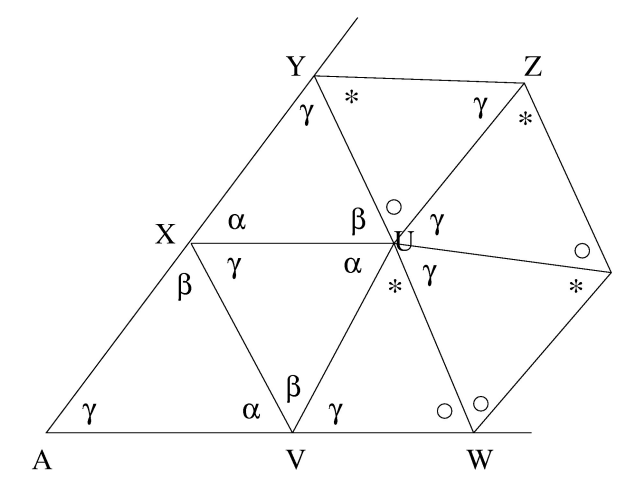

Fig. 18. Part of a dissection from Lemma 3, case 3.

Theorem 5. In every perfect, prime and simplicial dissection of a triangle $T$ into at least three triangles similar to $\triangle$, the angles of $\triangle$ satisfy one of the following conditions (with a suitable permutation of $\alpha, \beta$ and $\gamma$ ):

1. $\sin ^{4} \gamma=\sin ^{3} \beta \sin \alpha, \beta=2 \pi-3 \alpha, \gamma=2 \alpha-\pi$,

2. $\sin ^{5} \gamma=\sin ^{3} \beta \sin ^{2} \alpha, \alpha=2 \gamma, \beta=\pi-3 \gamma$,

3. $\sin ^{5} \gamma=\sin ^{4} \beta \sin \alpha, \beta=2 \pi-4 \alpha, \gamma=3 \alpha-\pi$.

Proof. We know that a dissection in question contains a vertex $x \in V_{3}$ with $\operatorname{deg} x=4$ or $\operatorname{deg} x=5$. If $\operatorname{deg} x=4$ then, with a suitable permutation of $\alpha, \beta$ and $\gamma, 3 \alpha+\beta=2 \pi$ because the angles that form a full angle around $x$ cannot be otherwise. Moreover, the angles marked by "*” (or the angles marked by "o") in Fig. 19 are equal, say they are equal to $\gamma$ (if not, then at least two triangles are congruent). Thus, $\alpha, \beta, \gamma$ satisfy the first condition. It can be easily checked that it has only one solution (see Fig. 2).

If $\operatorname{deg} x=5$, then the angles around $x$ can be the following (with a suitable permutation of $\alpha, \beta$ and $\gamma$ ): (a) $3 \alpha+\beta+\gamma=2 \pi$ or (b) $3 \alpha+2 \beta=2 \pi$ or (c) $4 \alpha+\beta=2 \pi$. In case (a) $\alpha=\pi / 2$, hence, three of five angles at the middle of the pentagon are right. Thus, the dissection contains two right triangles that share a leg, so it cannot be perfect as well as prime and simplicial. Moreover, the angles marked by " $*$ " (or the angles marked by "o") in Fig. 20 are equal. Thus, one of the two remaining conditions of the theorem must occur. It can be easily checked that each of them has exactly one solution.

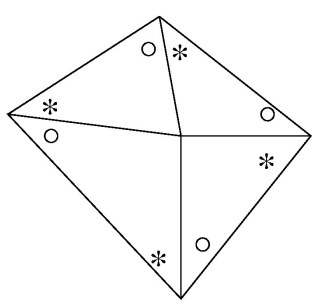

Fig. 19. Quadrangle induced by $x \in V_{3}$ with $\operatorname{deg} x=4$. 


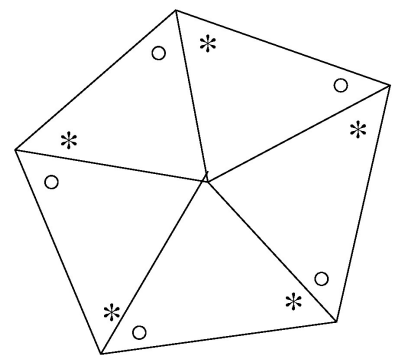

Fig. 20. Pentagon induced by $x \in V_{3}$ with $\operatorname{deg} x=5$.

\section{References}

1. R. L. Brooks, C. A. B. Smith, A. H. Stone and W. T. Tutte, The dissection of rectangles into squares, Duke Math. J. 7 (1940), 312-340.

2. A. J. W. Duijvestijn, Simple perfect squared square of lowest order, J. Combin. Theory Ser. B 25 (1978), 260-263.

3. P. J. Federico, Squaring rectangles and squares-a historical review with annotated bibliography, in Graph Theory and Related Topics (A. J. Bondy and U. S. R. Murty, eds.), pp.173-196, Academic Press, New York, 1979.

4. R. W. Freese, A. K. Miller, and Z. Usiskin, Can every triangle be divided into $n$ triangles similar to it?, Amer. Math. Monthly 77 (1970), 867-869.

5. H. Kaiser, Perfekte Dreieckszerlegungen, Elem. Math. 46 (1991), 106-111.

6. M. Laczkovich, Tilings of triangles, Discrete Math. 140 (1995), 79-94.

7. Z. Moroń, O rozkladzie prostokatów na kwadraty (in polish), Wiadom. Mat. I, 1 (1925), 75-94.

8. S. L. Snover, C. Waiveris and J. K. Williams, Rep-tiling for triangles, Discrete Math. 91 (1991), 193-200.

9. A. Soifer, How Does One Cut a Triangle? Center for Exellence in Mathematical Education, Colorado Springs, 1990

10. R. Sprague, Beispiel einer Zerlegung des Quadrats in lauter verschiedene Quadrate, Math. Z. 45 (1939), 607-608.

11. W. T. Tutte, The dissection of equilateral triangles into equilateral triangles, Math. Proc. Cambridge Philos. Soc. 44 (1948), 463-482.

Received December 16, 2003, and in revised form September 2, 2004 and November 17, 2004.

Online publication March 18, 2005. 